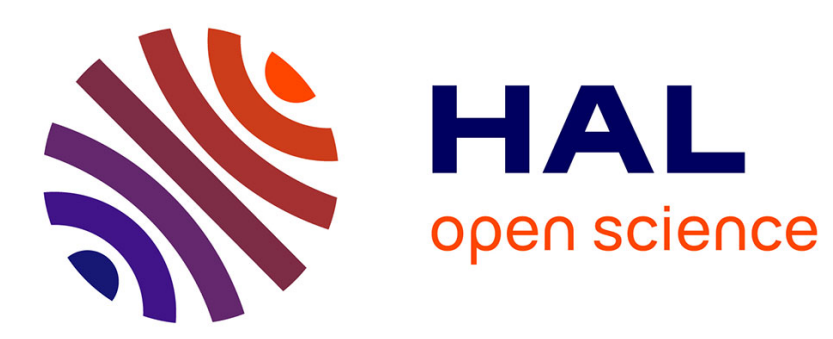

\title{
Culture de la rationalisation chez les ingénieurs durant la seconde industrialisation
}

Michel Letté

\section{To cite this version:}

Michel Letté. Culture de la rationalisation chez les ingénieurs durant la seconde industrialisation. 2010. halshs-00541840

\section{HAL Id: halshs-00541840 \\ https://shs.hal.science/halshs-00541840}

Preprint submitted on 1 Dec 2010

HAL is a multi-disciplinary open access archive for the deposit and dissemination of scientific research documents, whether they are published or not. The documents may come from teaching and research institutions in France or abroad, or from public or private research centers.
L'archive ouverte pluridisciplinaire HAL, est destinée au dépôt et à la diffusion de documents scientifiques de niveau recherche, publiés ou non, émanant des établissements d'enseignement et de recherche français ou étrangers, des laboratoires publics ou privés. 


\section{CULTURE DE LA RATIONALISATION CHEZ LES INGENIEURS DURANT LA SECONDE INDUSTRIALISATION}

Avant qu'il ne désigne un mode d'organisation de la production industrielle, du travail et de l'entreprise au sortir de la Première guerre mondiale, le terme «rationalisation » est une catégorie de la pensée et de l'action dont s'était largement emparée la classe des ingénieurs au cours du XIX ${ }^{\mathrm{e}}$ siècle. On retracera brièvement l'histoire de la formation et de la diffusion des cultures aux origines d'un mouvement qui culmine avec la seconde industrialisation en faveur de la planification des méthodes de gouvernement et d'une affirmation de la puissance publique dans le domaine de la production scientifique et de la pratique industrielle.

Dans la continuité du scientisme triomphant, de la consolidation de l'État et du rôle des compétences technico-administratives au cours de la $\mathrm{III}^{\mathrm{e}}$ République, quelques ingénieurs, tel Henry Le Chatelier, corpsard des Mines occupant les plus hautes fonctions académiques, incarne l'une de ces évolutions de la rationalisation vers la recherche des moyens de sa réalisation. Défenseur d'une science industrielle comme principe de rationalisation des rapports entre la science et l'industrie, il devient à la fin de sa carrière l'une des figures emblématiques de l'organisation scientifique du travail, le propagandiste zélé du taylorisme et du culte de la rationalisation.

\section{Unification culturelle sous l'égide de la rationalisation.}

Les ingénieurs du corps des Mines partagent au $19^{\mathrm{e}}$ siècle, avec beaucoup d'autres acteurs collectifs, le désir d'édifier une société future sur des bases rationnelles qu'anime un imaginaire fécond de l'efficacité industrielle ${ }^{1}$. Idéologies et doctrines alimentent et justifient dans cette perspective la transformation des structures sociales et productives, la recherche des conditions culturelles d'un consentement à l'industrialisation. Ils mobilisent des critères de rationalité spécifiques dont les fondements sont à rechercher tant dans la formation qu'ils ont reçu de leurs écoles respectives que dans l'exercice de leur activité professionnelle, mais aussi dans une attitude intellectuelle qui fait du progrès scientifique et technique le seul horizon légitime du processus inéluctable de rationalisation des sociétés modernes, au sens ou l'entend Max Weber au début du XX $\mathrm{XX}^{\mathrm{e}}$ siècle. Agents autoproclamés de la rationalisation, les ingénieurs contribuent à la promotion du développement industriel par leur action et la production d'une abondante littérature, se chargent de déterminer les moyens non seulement pratiques mais tout autant culturels et politiques de sa justification.

Les ingénieurs des Mines, ceux des autres corps et les autres agents de la rationalité technique, n'ont ainsi cessé de renforcer leur situation par rapport aux entités sociales, économiques et politiques de leur temps ${ }^{2}$. En partie héritiers du rationalisme des Lumières, mais dont ils détournent les principes aux fins d'une sécularisation de l'action politique et sociale, les ingénieurs contribuent au passage de la société d'ordres à la société de classes, de la sélection par la naissance à la désignation par le mérite et les capacités. La primauté du corps social et organique sur celle de l'individu libre et autonome, celle des devoirs collectifs sur celle des droits individuels, l'exaltation de la collectivité soumise aux lois naturelles et mécaniques de son destin figurent parmi les registres de la pensée auxquels se réfèrent plus volontiers des ingénieurs en quête de reconnaissance et de justification de leur autorité. Certes l'enseignement technique qu'ils ont reçu leur a inculqué le respect de la hiérarchie, de l'ordre et de la discipline dans l'entreprise, mais c'est surtout à l'autorité de la rationalité scientifique et technique à laquelle ils se réfèrent, autorité dont ils s'affirment comme les dépositaires et les garants

\footnotetext{
${ }_{1}^{1}$ Antoine Picon, «Imaginaires de l'efficacité, pensée technique et rationalisation », Réseaux, 109, 2001, pp. 18-50.

${ }^{2}$ André Thépot dir., L'ingénieur dans la société française, Paris, Editions ouvrières, 1985, 329 p. ; André Grelon, «Les ingénieurs, encore », Culture technique, 12, 1984, p. 11-17 ; Maurice Levy-Leboyer, "The contribution of french scientists and engineers to the development of modern managerial structures in the early part of the twentieth century », dans Science technology and society in the time of Alfred Nobel, The Nobel Foundation, Oxford, Pergamon Press, 1982, p. 283-297; François Perroux, Industrie et création collective. Saint Simonisme du XX siècle et création collective, Paris, Presses universitaires de France, 1964, 204 p. ; Jean Meynaud, «Les techniciens et le pouvoir », Revue française de science politique, 1, 1957, p. 5-37.
} 
légitimes ${ }^{3}$. L'ordre rationnel invoqué s'applique dès lors à tous les domaines de l'organisation des institutions humaines et détermine leur fonctionnement optimal. La logique est celle d'une rationalité par rapport à une fin : le rendement maximum, la production efficace, la performance des institutions.

Partagé entre une fonction technique et une fonction sociale, l'ingénieur bouscule hiérarchies et valeurs traditionnelles, abandonne ses origines aristocratiques et élitistes à l'ancienne pour en inventer de nouvelles, plus ancrées dans la révolution scientifique, la compréhension et la formulation de lois universelles, indiscutables et non négociables auxquelles doivent nécessairement se soumettre les masses irrationnelles sous peine de se voir refuser l'exercice légitime de leur raison. La classe des ingénieurs s'est affirmée à la fin du $19^{\mathrm{e}}$ siècle comme celle de techniciens de gouvernement dont l'ambition est d'articuler au sein d'un même système efficace la production et la question sociale ${ }^{4}$. Les ingénieurs supportent au siècle suivant le projet d'une organisation des relations industrielles et de la collectivité sociale selon des modalités qu'autorise une culture de la rationalisation forgée par un siècle de luttes pour leur professionnalisation et la reconnaissance de leur statut ${ }^{5}$.

\section{La question sociale et l'ingénieur}

L'irruption des masses à la fin du $19^{\mathrm{e}}$ siècle dans la production et la participation politique confronte les ingénieurs à des questions nouvelles. Celle d'abord de la légitimité de la prise de décision en matière d'organisation de l'industrie, de conduite de la production, de direction des entreprises, des structures de commandement, de pertinence d'une évolution de la législation sociale et du travail. Quelle place les ingénieurs pensent-ils devoir tenir dans une société industrialisée où la démocratie libérale, l'opinion publique, le mouvement ouvrier et les rapports de force prennent une importance croissante ? Quelles stratégies ont-ils déployé en sorte d'assurer la reconnaissance de leur rôle et de leur statut?

Dans la mouvance de l'Union sociale des ingénieurs catholiques ou de la Société d'économie sociale, de la Société des ingénieurs civils ou encore du Musée social, la réception de la question sociale évolue vers la recherche des moyens techniques de gouvernement des populations. L'un des rôles que s'octroie l'ingénieur est celui de pourvoir aux besoins des pratiques de gouvernement du social par le recours à la science, à la méthode expérimentale et l'observation des faits ${ }^{6}$. L'ingénieur, trait d'union entre les intérêts contradictoires de l'industriel et de l'ouvrier, acteur de la paix sociale et adepte du paternalisme patronal, s'affirme dès lors comme un personnage central de la seconde industrialisation, le partisan d'une discipline des agents de l'économie sous l'égide de la rationalisation. Exaltée par Émile Cheysson dans un texte bien connu de 1897, la science doit donc avoir, écrit-il, sa place marquée pour l'outillage social, comme elle l'a pour l'outillage industriel' L'ingénieur est ainsi dans la tradition conservatrice et leplaysienne appelé à constituer le noyau d'une aristocratie nouvelle, une autorité sociale dont l'ambition ou la prétention est de transcender les divisions politiques et les divergences d'intérêts entre syndicats et patronat, d'assurer le lien entre employeurs et employés, patrons et ouvriers ${ }^{8}$. Il endosse la responsabilité de l'expert neutre, tendu vers

\footnotetext{
${ }^{3}$ Michel Bouillé, Enseignement technique et idéologique au XIX ${ }^{e}$ siècle, Thèse, 1972, 490 p.

${ }^{4}$ Antoine Picon, L'invention de l'ingénieur moderne. L'École des ponts et chaussées, 1747-1851, Paris, Presses de l'École des ponts et chaussées, 1992, 768 p. ; François Vatin, Morale industrielle et calcul économique dans le premier XIX ${ }^{e}$ siècle. L'économie industrielle de Claude-Lucien Bergery (1787-1863), Paris, L'Harmattan, 2007, 411 p.

${ }^{5}$ André Grelon, dir. Les ingénieurs de la crise. Titre et profession entre les deux guerres, Paris, Éditions de l'Ehess, 1986, 461 p. ; André Thepot, dir. L'ingénieur dans la société française, Paris, Éditions ouvrières, 1985, 332 p.

${ }^{6}$ Rémi Baudouï et Yves Cohen, dir. Les chantiers de la paix sociale (1900-1940), Fontenay - Saint Cloud, ENS éditions, 1995, $331 \mathrm{p}$.

${ }^{7}$ Émile Cheysson, «Le rôle social de l'ingénieur », La réforme sociale, 1897, p. 21-35. Parmi d'autres écrits du genre qui se multiplient durant la seconde industrialisation: Maurice Bellom, «L'Enseignement économique et social à l'École nationale supérieure des mines. Le rôle économique et social de l'ingénieur », Le Génie civil, 1906, 31 p. ; Léon Foulon, «Le Rôle social de l'ingénieur moderne », Revue industrielle de l'Est, 1908, p. 1-9; Edouard Maurel, L'ingénieur social dans l'industrie, Paris, Librairie du « Recueil Sirey », 1929, 285 p. ; George Lamirand, Le rôle social de l'ingénieur : scènes de la vie d'usine, Paris, Plon, 1954, $301 \mathrm{p}$.

${ }^{8}$ Renzo Gubert et Luigi Tomasi (dir.) Le catholicisme social de Pierre Guillaume Frédéric Leplay, Milan, FrancoAngeli, 1994, 134 p. ; Françoise Arnault, Histoire de Frédéric Le Play, de la métallurgie à la science sociale, Nancy, Presses universitaires de Nancy, 1993, 252 p.; André Thepot, «Les ingénieurs des mines, le patronat et la seconde
} 
l'optimisation des moyens de la production au bénéfice de tous. La neutralité et la compétence scientifique promettaient ainsi de dépasser la lutte des classes et d'orienter la communauté des producteurs vers la recherche du bien commun : l'efficacité, le rendement accru et un meilleur revenu pour tous, que ce soit sous la forme de profits ou de salaires.

Cette façon de concevoir leur contribution à la réforme sociale par la rationalisation se repère chez nombre d'ingénieurs qui ont participé, à des titres divers, aux actions de la nébuleuse réformatrice et de ses réseaux au tournant des $19^{\mathrm{e}}$ et $20^{\mathrm{e}}$ siècles$^{9}$. Dans le domaine de la législation du travail notamment, où Arthur Fontaine figure au rang des acteurs emblématiques parmi les ingénieurs des Mines $^{10}$. Mais d'autres ont, avec ou comme lui, investi de nombreux autres champs de la réforme sociale et différents lieux de la sociabilité scientifique, industrielle et politique dans une même recherche des moyens de l'action: académies, alliances, associations, comités et commissions, sociétés d'économie sociale, congrès, écoles, fédérations, offices, unions et revues qui se sont imposés comme autant d'espaces où se débattent et s'élaborent les termes de la réforme sociale, l'exploration des possibles que permettrait la rationalité instrumentale ${ }^{11}$. La production des savoirs scientifiques y prend une part des plus importantes, désignée à la fois comme un moyen de réalisation de la rationalisation, mais aussi comme un schéma d'action en politique ${ }^{12}$. La conviction que la science positive et la rationalité technique se doivent de déterminer la marche des sociétés modernes, jusque et y compris en ce qui concerne les registres de la politique et du social est cependant des plus courantes. Parmi d'innombrables expressions de cette conviction devenue banalité au tournant du siècle, le sénateur Jean-Honoré Audiffred préconise par exemple en 1911 de recourir systématiquement à la méthode expérimentale et de placer la science au dessus de la politique pour gérer la démocratie et l'instabilité de l'opinion publique ${ }^{13}$. La préoccupation reste de désigner les experts légitimes en charge de la prise de décision devant s'appliquer à l'ensemble de la population, et en dernière instance de déterminer les modalités d'une gestion optimale des intérêts de tous par l'organisation rationnelle de la société industrielle. Une conception radicale du rôle de la science dans la conduite des sociétés humaines s'impose, avec cette idée qu'elle doit assurer désormais les fonctions d'un arbitrage en matière de politique et du social.

\section{Rationalisation et centralisme technocratique.}

La rationalisation prend en France le sens surtout d'une application des principes et des méthodes scientifiques à l'organisation du travail et de la production ${ }^{14}$. Elle est essentiellement une affaire d'ingénieurs, animés par une idéologie d'inspiration scientiste, convaincus de devoir s'imposer comme un groupe social dont la mission est d'œuvrer à l'extension des critères de la rationalité technique aux domaines, non seulement de la production, mais aussi de l'organisation sociale. Si la rationalisation de la production se situe au point de conjonction de l'économie, de la technique et du

industrialisation », dans Maurice Levy-Leboyer (dir.), Le patronat de la seconde industrialisation, Les Cahiers du mouvement social, 4, 1979, p. 237-246.

${ }^{9}$ Christian Topalov, dir. Laboratoires du nouveau siècle. La nébuleuse réformatrice et ses réseaux en France, 1880 1914, Paris, Éditions de l'Ehess, 1999, 574 p. ; Janet Horne, Le Musée social. Aux origines de l'État providence, Paris, Belin, 2004, 384 p. ; Alain Chatriot, Odile Join-Lambert et Vincent Viet (dir.), Les politiques du travail (1906-2006) : acteurs, institutions, réseaux, Rennes, Presses universitaires de Rennes, 2006, 518 p.

${ }^{10}$ Michel Cointepas, Arthur Fontaine, 1860-1931 : un réformateur, pacifiste et mécène au sommet de la troisième république, Rennes, Presses Universitaires de Rennes, 2008, 382 p. ; Isabelle Lespinet-Moret, L'Office du travail (18911914) : la République et la réforme sociale, Presses universitaires de Rennes, 2007, 370 p.

${ }^{11}$ Alain Chatriot, "Réformer le social sous la Troisième République », Revue d'histoire moderne et contemporaine, 56, 2009 , p. $40-53$.

12 Michel Pinault, La science au Parlement. Les débuts d'une politique des recherches scientifiques en France, Paris, éditions du CNRS, 2006, 159 p.

${ }^{13}$ Henri Audiffred, «La réforme administrative. Organisation régionale de la France. La méthode expérimentale en politique », Revue politique et parlementaire, 67, 1911, p. 425-445.

${ }_{14}$ A. Moutet, Les logiques de l'entreprise ... op. cit. et «Ingénieurs et rationalisation en France de la guerre à la crise (1914-1929) », dans André Thépot, dir. L'ingénieur dans la société française, Paris, Les éditions ouvrières, 1985, p. 71-108 ; Odette Hardy-Hémery, «Rationalisation technique et rationalisation du travail à la Compagnie des Mines d'Anzin (19271938) », Le Mouvement social, 72, 1970, p. 3-48 ; Thierry Kirat, "Taylorisme et rationalisation du travail en France et en Allemagne (1919-1939) », Revue d'Economie Politique, 100, 1990, p. 58-82. 
social, sa signification est aussi profondément politique et culturelle $\mathrm{e}^{15}$. L'exaltation de la rationalisation de la production dans l'entre-deux-guerres en est le point d'orgue, l'expression d'une volonté ferme de redéfinir le rôle des acteurs sociaux et des agents de l'économie. Elle s'était cependant imposée bien avant le premier conflit mondial ${ }^{16}$. Ce dernier a toutefois autorisé les tentatives de réalisations concrètes, notamment dans le domaine de l'administration des relations entre l'État et l'économie industrielle ${ }^{17}$. Rationalisation et bureaucratisation sont organiquement liées, non seulement en France où cette articulation prend la forme singulière d'une centralisation technocratique quand il est question de gérer la société de masse, mais partout ailleurs parmi les sociétés industrielles ${ }^{18}$. André Thépot montre ainsi comment, dans un État centralisateur comme la France, les ingénieurs des Mines ont progressivement pris une place nécessairement importante ${ }^{19}$.

Une des manifestations remarquables du centralisme technocratique a été la remise au gouvernement en 1919 par Étienne Clémentel, ministre du commerce et de l'industrie durant la Première guerre mondiale, d'un rapport général sur l'industrie française où sont exposées les modalités d'organisation de la production qu'il faudrait désormais selon lui mettre en œuvre ${ }^{20}$. Ce document d'histoire économique de la France en guerre est aussi un matériau pour l'histoire politique, intellectuelle, sociale et culturelle de la III $^{\mathrm{e}}$ République, et plus précisément de l'un de ses épisodes technocratiques les plus intenses ${ }^{21}$. Le rapport d'Étienne Clémentel s'inscrit en effet dans une série de travaux qui témoignent une volonté ferme d'intervention de l'administration pendant les hostilités et de la façon dont les autorités publiques pensent devoir discipliner l'économie de guerre sous l'égide de la rationalisation et de sa conduite par les ingénieurs. L'entreprise marque en tout cas l'évolution d'une pensée et de l'action gouvernementale. Au-delà des chiffres et de leur rhétorique s'expriment en effet des enjeux politiques, des justifications de réforme des cadres d'intervention de l'État, une volonté de renégocier les termes de la question sociale, l'affirmation d'une culture de gouvernement qui s'élabore au fil de l'action. Ce document monumental s'avère au final exprimé clairement le désir d'édifier une économie rationnelle qu'appellent de leurs vœux les partisans d'une technocratie, et auxquels le Ministre Étienne Clémentel s'est allié pour la circonstance.

${ }^{15}$ Charles S. Maier, «Between Taylorism and Technocracy : European ideologies and the vision of industrial productivity in the 1920s », Journal of contemporary history, 5, p. 27-61 ; Lyndall. F. Urwick, "The rationalization movement", dans Leslie Hannah, dir. The rise of the corporate economy, Londres, John Hopkins university press, 1976, p. 29-44 et The meaning of rationalisation, Londres, Nisbet, 1929, $160 \mathrm{p}$.

${ }^{16}$ Yves Cohen, «Le vingtième siècle commence en 1900. Sciences, technique, action », Alliage, 20-21, 1994, p. 88-104 ; Patrick Fridenson, «Un tournant taylorien de la société française (1904-1918) », Annales ESC, 42, 1987, p. 1031-1060.

${ }^{17}$ Richard F. Kuisel, Le capitalisme et l'État en France. Modernisation et dirigisme au XX siècle, Paris Gallimard, 1981, 477 p.; Stéphane Rials, Administration et organisation, 1910-1930. De l'organisation de la bataille à la bataille de l'organisation dans l'administration française, Paris, Beauchesne, 1977, 271 p. ; Paul Rabinow, French modern : norms and forms of the social environment, Chicago, University of Chicago press, 1995, $454 \mathrm{p}$.

${ }^{18}$ Rolph Torstendahl, Bureaucratisation in Northwestern Europe, 1880-1985: domination and governance, Taylor \& Francis, 1991, 339 p. ; Adrian Rossiter, Experiments with corporatist politics in republican France, 1916-1939, Oxford, Thesis Nufflied College, 1986, 357 p. ; George G. Humphreys, Taylorism in France, 1904-1920 : the impact of scientific management on factory relations and society, New York, London, Garland, 1986, 275 p. ; John F. Godfrey, Capitalism at War : Industrial policy and bureaucracy in France, 1914-1918, Berg Publishers Ltd, 1987, 313 p.; Charles S. Maeir, Recasting bourgeois Europe: stabilization in France, Germany, and Italy in the decade after World War I, Princeton University Press, 1975, $650 \mathrm{p}$.

${ }^{19}$ André Thépot, «Les ingénieurs du corps des Mines. Évolution des fonctions des ingénieurs d'un corps d'Etat au $19^{\mathrm{e}}$ siècle », Culture Technique, 12, 1984, p. 55-61.

${ }^{20}$ Volumes disponibles à la Bibliothèque nationale de France sous la côte 4- LF262- 348 (1 à 3) : Ministère du Commerce, de l'Industrie, des Postes et Télégraphes, des Transports maritimes et de la Marine marchande. Direction des études techniques, Rapport général sur l'industrie française, sa situation, son avenir (d'après les travaux des sections du Comité consultatif des arts et manufactures et de la Direction des études techniques). Première partie. Étude de la situation des principales industries avant la guerre et de leur expansion possible (vol. 1 et 2); Deuxième partie : Les Méthodes d'expansion économique (vol. 3), Paris, imprimerie Nationale, 1919.

${ }_{21}$ Michel Letté, «Le rapport d'Étienne Clémentel (1919): l'avènement administratif des technocrates et de la rationalisation », Documents pour l'histoire des techniques, 20, décembre 2010 (à paraître) ; Clothilde Druelle-Korn, Un laboratoire réformateur, le Département du commerce en France et aux États-Unis de la Grande Guerre aux années vingt, Paris, Thèse IEP, 2004, 596 p.; Yves Roussel, De la science et de l'industrie ; Ministère Clémentel, 1915-1919, DEA de l'EHESS, 1985, 53 p. ; Fabienne Bock, Un parlementarisme de guerre, 1914-1919, Paris, Belin, 2002, 350 p. et «L'exubérance de l'État en France de 1914 à 1918 », Vingtième Siècle, 3, 1984, p. 41-52; George-Henri Soutou, L'or et le sang. Les buts de guerre économiques de la Première guerre mondiale, Paris, Fayard, 1989, 963 p. ; Marc Trachtenberg, « "A new Economic Order" : Étienne Clémentel and French Economic Diplomacy during the first world war», French Historical Studies, 10 (2), 1977, p. 315-341. 
L'emprise administrative de l'État sur l'économie n'est cependant pas ce qui le préoccupe le plus. Son Rapport général sur l'industrie française, sa situation, son avenir, qui dès la première ligne devient le «rapport général sur l'Organisation de la Production nationale au lendemain de la guerre » (notez les termes affublés d'une majuscule), annonce d'emblée et précise ce dont il est véritablement question: la mise en œuvre d'un programme de réorganisation rationnelle de la production industrielle. Le salut de l'économie française passe par l'organisation, dont la mention apparaît une vingtaine de fois dans l'introduction, associée aux termes «doctrine », «principe » et «programme ${ }^{22}$. La France a besoin d'une ligne de conduite, d'un guide pour l'action, et plus encore comme l'indique plus loin Étienne Clémentel, d'une doctrine économique. Le rapport est défini par son auteur comme celui d'un comité d'experts qui indiquent les moyens à mettre en œuvre pour relever la France. Ce programme de gouvernement soumis au Parlement se présente sous la forme d'une série d'avis qui se veulent solidement établis sur la base d'études scientifiques et techniques. Ils s'énoncent dès lors comme des solutions nécessairement valides et légitimes, et que les politiques se doivent de considérer le plus sérieusement du monde.

Ce rapport n'est en aucun cas le plaidoyer pour une étatisation de l'économie. Étienne Clémentel n'y a en réalité jamais songé au motif que la guerre l'imposait. L'état d'esprit qui préside à l'élaboration de son programme est bien au contraire de se prémunir d'une remise en cause trop radicale de l'ordre économique libéral d'avant-guerre. Certes il juge comme une évidence de devoir administrer, orienter, encadrer l'économie, mais certainement pas de condamner son régime antérieur au profit d'une emprise totalisante de l'État. Sa proposition doit plutôt être considérée comme la crainte de ce qu'Étienne Clémentel considère comme la véritable menace : celle du collectivisme où l'État voit ses prérogatives s'élargir sans limites. Le programme suggéré vise en fait à échapper à l'alternative du libéralisme traditionnel et du socialisme, tout en veillant à préserver l'ordre libéral et ses valeurs, à pérenniser un système social et économique d'avant-guerre qu'Étienne Clémentel défend sur le plan intellectuel et politique. Il revendique donc la légitimité d'un régime libéral organisé sur des bases scientifiques comme le seul capable d'assurer la prospérité du pays, à condition de l'aménager et d'agir sur ce qu'il considère comme les travers français de la production industrielle, sur l'indiscipline de ses agents, de confier aux producteurs et aux élites dirigeantes la conduite des initiatives collectives.

Le rapport d'Étienne Clémentel doit donc être lu comme la quête d'une doctrine visant la transformation de l'économie libérale qui l'éloignerait du spectre socialiste, mais aussi de l'emprise des masses et de l'opinion publique. Ce n'est ni le socialisme que propose Étienne Clémentel, ni le libéralisme économique orthodoxe, mais bien la mise au travail, la mise en conformité de l'organisation et du gouvernement rationnel des agents économiques avec les impératifs du productivisme. La rationalisation comme instrument d'une domination des élites a dans ce cas pour fonction d'opposer au collectivisme révolutionnaire et au socialisme un contre-modèle faisant de l'intérêt collectif opposé à l'individualisme l'objectif de sa politique. Le «collectivisme rationnel » permet selon lui de canaliser les prétentions démocratiques des masses par la quête d'une politique conciliatrice entre les tenants de la conservation libérale, de la réforme sociale et les aspirations socialistes de la classe ouvrière. Ce qui se met en place durant la guerre s'inscrit dès lors dans la continuité d'une tradition élitiste et non démocratique, avec pour horizon l'objectivation des modes d'organisation et d'administration par l'État d'une économie orientée par les experts et la mise en œuvre technique par les producteurs.

Les injonctions à la rationalisation de l'économie invitent dès lors à l'instauration d'un régime gestionnaire et technocratique des affaires industrielles et sociales. Elles recommandent un nouvel ordre où la promotion d'une culture de la science et de la technique, de la production de savoirs mis au service de la prise de décision rationnelle, débouchent inéluctablement sur l'adhésion des travailleurs au productivisme et à la promesse d'une croissance continue des richesses à partager. Cette revendication d'une économie s'érigeant sur la neutralité de connaissances objectives permet ainsi, en la technicisant, d'échapper en partie à la décision politique et à la critique individuelle. Ainsi le projet

\footnotetext{
${ }^{22}$ Les principales occurrences sont logiquement «industrie » (71), «production » (60) et « commerce» (59). Les termes ou les expressions renvoyant au domaine administratif (réforme, gouvernement, administration, ministère, contrôle, enquête et études) totalisent 40 mentions. «Capital » et «capitaux » rassemblent 21 occurrences, «travail » 11, mais « démocratie » et «social » ne sont mobilisés respectivement que 1 et 2 fois sur la totalité du texte.
} 
pérennise les principes traditionnels d'une $\mathrm{III}^{\mathrm{e}}$ République autoritaire où la puissance, la performance et la dynamique de perfectionnements permanents justifient la prise en charge par les élites techniques et expertes des orientations de l'organisation économique et de la réforme sociale ${ }^{23}$.

De ce point de vue, le rapport d'Étienne Clémentel peut être considéré comme la manifestation d'une emprise technocratique, un matériau pour l'histoire de ce qui sera un peu plus tard désigné par «capitalisme d'État» ou «économie administrée ». Il est aussi un matériau pour l'histoire de ces élites qui ont en charge la conduite de l'économie dans les années 1920 : les industriels toujours, mais surtout les technocrates, les experts, et les scientifiques qui trouvent l'opportunité de consolider des liens noués avant-guerre sur la base d'une montée en rationalité de l'organisation de la société industrielle ${ }^{24}$. La politique et l'action d'Étienne Clémentel s'inscrivent dès lors dans la perspective d'une construction entre les deux guerres de systèmes de direction et de management de l'économie sous la houlette du corporatisme d'État et de la technocratie, légitimant ses options par l'apolitisme des experts et de leurs avis. Comme les penseurs classiques et leurs épigones qui reformulent la théorie économique libérale dans les années 1870, Étienne Clémentel témoigne de sa foi dans l'existence de lois quasi naturelles en matière d'économie industrielle.

La guerre a ainsi autorisé la réalisation consensuelle de l'utopie technocratique pour la gestion de l'économie et du social où l'adhésion volontaire à l'autorité de la science et de la technique détermine des choix politiques, guide le comportement rationnel des agents économiques. Étienne Clémentel se fait à cette occasion l'avocat, peut-être non de la technocratie elle-même, ou même de l'économie dirigée, mais au moins - comme il le dit lui-même - de l'économie « orientée par l'État ». Il apparaitt pourtant bien comme un chef de file parmi les élites républicaines d'obédience technocratique. Elles s'affairent à stabiliser l'ordre social par la promotion de la rationalisation, ou du moins en en créant les conditions. Nul doute que la guerre et sa promesse d'une conclusion victorieuse au prix d'une réorganisation rationnelle de la société industrielle et de sa délégation aux technocrates auront contribué à édifier, avec Étienne Clémentel, la perspective d'un avenir suffisamment optimiste et crédible pour déclencher les actions qui engendreraient sa propre réalisation. L'idée s'est répandue d'une nécessaire délégation à l'État et à ses scientifiques, ses techniciens, ses ingénieurs qui acquièrent dès lors toute légitimité pour prévoir, organiser et agir en matière d'économie sous l'égide de la rationalisation.

Étienne Clémentel aura créé pour les compétences techniques et les experts l'opportunité d'une consolidation de leurs rôles au sein des instances gouvernementales, des commissions, comités et offices dédiés. Il aura consolidé l'autonomie de l'administration par rapport au gouvernement ${ }^{25}$. C'est en tout cas ce que les technocrates cultivent dans le giron d'Étienne Clémentel et tentent de prolonger en invoquant l'impératif de poursuivre en temps de paix, et sur le modèle des actions entreprises lors $\mathrm{du}$ conflit, la rationalisation de l'État et de ses pratiques en matière d'économie. Ce vœu d'institutionnaliser leur fonction politique s'exprime clairement dans le rapport de 1919, notamment par la proposition d'instaurer un Conseil d'État Technique sans lequel les parlementaires et le législateur ne pourraient arrêter de décision sans une consultation préalable. Constitué d'experts scientifiques et de techniciens, cette instance serait l'une des bases du dirigisme technocratique tendant vers la rationalisation de la politique.

En définitive, les travaux de l'équipe d'Étienne Clémentel ont constitué une sorte de laboratoire de la technocratie politique et sociale. Son histoire se prolonge assurément dans l'entre-deux guerres, la rationalisation devenant contagieuse. D'autres cadres de pensée s'affirment. Ils permettent au

\footnotetext{
${ }^{23}$ Gérard Brun, Technocrates et technocratie en France (1914-1945), Paris, Éditions Albatros, 1985, 324 p. ; Dominique Janicaud, La puissance du rationnel, Paris, Gallimard, 1985, 386 p. ; Jean Meynaud, Technocratie : mythe ou réalité ?, Paris, Payot, 1964, 297 p.

${ }^{24}$ Aimée Moutet, Les logiques de l'entreprise ... op. cit.; George G. Humphreys, Taylorism in France, 1904-1920 : the impact of scientific management on factory relations and society, New York, London, Garland, 1986, 275 p. ; P. Fridenson, «Un tournant taylorien ... », op. cit.; Charles S. Maier, « Entre le taylorisme et la technocratie : idéologies et conceptions de la productivité industrielle dans l'Europe des années 1920 », dans Lion Murard et Patrick Zylberman (dir.) Le Soldat du travail : guerre, fascisme et taylorisme, Recherches 32/33, 1978, p. 95-134 ; Lyndall F. Urwick, "The rationalization movement », dans Leslie Hannah (ed.), The rise of the corporate economy, London, J.H. University Press, 1976, p. 29-44 et The Meaning of Rationalisation, London, Nisbet, 1929, $160 \mathrm{p}$.

${ }^{25}$ Francine Soubiran-Payet, «Parlement, administrateurs et experts (1900-1914). Le discours de la compétence », Vingtième Siècle, 93, 2007, p. 151-163; Alain Chatriot, «Les offices en France sous la Troisième République. Une réforme incertaine de l'administration », Revue française d'administration publique, 120, 2006, p. 635-650.
} 
monopole de la rationalité instrumentale de s'exercer sur la raison et l'autonomie individuelles, à la rationalisation de devenir l'apanage des techniques de gouvernement des agents économiques et des populations.

\section{La rationalisation chez Henry Le Chatelier.}

Parmi les principaux instigateurs et animateurs de l'entreprise d'Étienne Clémentel, figure le corpsard Henry Le Chatelier. C'est ce dernier qui aura en réalité imposé aux travaux du ministère du commerce sa dimension la plus technocratique.

De la fin des années 1870, début de sa carrière, jusqu'au conflit mondial à l'issue duquel une impulsion déterminante est donnée à la structuration des liens entre la communauté scientifique et celle des producteurs, Henry Le Chatelier élabore ce qu'il désigne par la "science industrielle », notion développée en guise de discipline embrassant toute forme d'activité vouée à la rationalisation ${ }^{26}$. Ainsi, bien avant qu'elle ne devienne une catégorie d'action, Henry Le Chatelier avait tenté de promouvoir un modèle de rationalisation de la question sociale par celle des rapports entre la production scientifique et la pratique industrielle. Il s'agissait non seulement d'exposer les connaissances utiles à l'industrie, mais encore d'aménager une zone intermédiaire entre «savoir » et "produire », dotée d'un schéma intellectuel spécifique, conçu comme un guide opérationnel mis au service des impératifs de la rationalisation de l'économie.

A l'âge de 69 ans, juste après la Première Guerre mondiale, Henry Le Chatelier entame sa retraite. Carrière et production scientifique s'arrêtent. Il se consacre alors à ses élèves et à ses disciples, anime une école, mais surtout développe ses réflexions qui prolongent les principes de la science industrielle. Il ne s'impose plus seulement comme un chercheur et un professeur, mais comme une autorité consacrée. Et c'est fort de sa réputation qu'il s'engage dans le débat sur l'extension d'une application sans limite de la science à l'ensemble des activités de la société. Il devient propagandiste zélé et caution scientifique du taylorisme qu'il s'efforce d'adapter au contexte français selon des vues toutes personnelles ${ }^{27}$. En fait, c'est comme une révélation qu'il a reçu au début du siècle les théories de l'ingénieur américain Frederick Taylor. Il les considère comme l'application juste et pertinente de la science industrielle au cas de la production et de l'économie suivant toutes ses composantes. Aussi il inscrit son nom sur la liste des théoriciens de l'organisation scientifique du travail. Plus généralement, il contribue au mouvement qui se précise de rationalisation de la production. Ses ambitions et ses prétentions scientistes s'étendent dès lors jusque et y compris aux domaines des relations sociales, de la formation, de l'économie politique et même de la morale.

Pour Henry Le Chatelier, la science industrielle incarne un type de rationalité qui en définitive débouche sur la démonstration qu'elle se définit elle-même comme science. L'adhésion à un système de critères rationnels appuyés sur la science et orientés vers l'économie conduit à la perspective d'une domination légitime, l'exercice d'un contrôle, à la fois du monde scientifique, industriel et de l'espace social. Elle recoupe le projet d'une transformation de l'environnement intellectuel et matériel. Autrement dit, la science industrielle sous-tend un projet de rationalisation. Elle désigne l'extension des domaines de la société soumis aux critères de décision rationnelle. Elle se traduit non seulement par l'institutionnalisation de l'activité scientifique, par l'accroissement de la production industrielle, mais encore par la mobilisation de la science et de la technique à une fin de légitimation de l'État, de sa consolidation, de son autonomie, de sa domination ${ }^{28}$.

Au-delà des divergences de conviction sur la nature de la liaison entre la production scientifique et la pratique industrielle, la rationalisation recouvre une série d'attitudes et de comportements communs. En conséquence, l'organisation rationnelle suppose une hiérarchie : quelques individus dotés des plus hautes compétences scientifiques dirigent une majorité d'exécutants pour la production d'objets standardisés d'une nature déterminée et imposent la méthode pour y parvenir. Tel est l'enjeu pour Henry Le Chatelier au travers de sa promotion de la science industrielle : refondre les fonctions du

\footnotetext{
${ }^{26}$ Michel Letté, Henry Le Chatelier (1850-1936) ou la science appliquée à l'industrie, Rennes, Presses universitaires de Rennes, 2004, $258 \mathrm{p}$

${ }^{27}$ Odile Henry, « Henry Le Chatelier et le taylorisme », Actes de la Recherche en Sciences Sociales, 133, 2000, p. 79-88.

${ }^{28}$ Bertrand Badie et Pierre Birbaum, Sociologie de l'État, Paris, Grasset, 1979, p. 38-50.
} 
savant, de l'industriel et de son intermédiaire privilégié, l'ingénieur, dans le cadre d'une programmation méthodique du progrès. Le projet permanent de rationalisation auquel invite la science industrielle précède l'entreprise de Henry Le Chatelier. Elle se rapporte dans ce cas à une conduite des acteurs de la production, conformément à des règles dictées par des critères rationnels parés de l'objectivité. Au-delà, elle est assimilée à un paradigme, compris comme la somme des convictions, valeurs et techniques partagées par la communauté des ingénieurs.

Dans l'univers rationalisé de Henry Le Chatelier, la science est une institution qui fournit la matrice à partir de laquelle se justifie l'action politique. Elle la légitime. Une politique scientifique doit œuvrer à la professionnalisation des liens entre science et société, ce qui façonne en retour une vision de l'État dans laquelle la science est convoquée pour ses méthodes et ses capacités organisationnelles présumées. La politique pour la science fait place à la politique par la science ${ }^{29}$. La science est alors "expression, symbole d'une visée totalisante ${ }^{30}$ ». Elle suggère une adéquation entre un système politique et un système scientifique, mais définissant de manière étroite les voies de la scientificité, ainsi que ses réalisations.

Un tel schéma de pensée s'alimente d'une attitude naturaliste et déterministe selon laquelle la science expérimentale est le modèle à suivre. Il s'appuie sur la capacité d'unification d'une science dédiée à la révélation des lois naturelles régissant le monde. Tout comme le matériel et l'organique semblent être gouvernés par des lois immuables, l'univers social serait, lui aussi, déterminé par des lois universelles qu'il s'agirait en toute circonstance de dévoiler. La démarche aboutit à la formulation, puis à la formalisation de lois sous la forme d'un langage et d'un mode d'exposition calqué sur le modèle des sciences de la nature. Il ne s'agit plus seulement de rendre compte scientifiquement de la réalité industrielle, mais d'intervenir d'une manière scientifique en faisant usage d'un corpus de connaissances objectivement élaborées et communicables ${ }^{31}$. L'entreprise scientifique, parce qu'elle propose un traitement rationnel et systématique du social, suggère une alternative politique, un modèle de conduite et un cadre pour l'action. Il est question d'une mise en science de la politique qui abouti à faire de son exercice plus une technique qu'un art. Le savoir objectif considéré comme le résultat de la démarche scientifique promet ainsi de ramener les conflits et leur gestion par les politiques publiques à un système de problèmes, de solutions et d'équations intermédiaires. Il prétend dépasser les clivages partisans. La science est posée comme archétype d'un système d'organisation humaine, de gestion rationnelle et d'administration des sociétés modernes. Elle impose un principe de discipline politique. En conséquence, Henry Le Chatelier cherche plus à atteindre la glorification de la science par l'État plutôt que la célébration de l'État par la science. La science est un instrument, devient un moyen d'action pour dompter le social, structurer l'économie, et allouer un but à l'État, qui est à la fois celui de sa propre affirmation et de sa puissance.

Dans le prolongement d'une volonté de régenter le monde par la rationalisation, Henry Le Chatelier confronte la science et la politique comme on oppose l'efficacité et le rationnel au gaspillage et au mensonge. La base de sa revendication reste l'utilitarisme qu'exprime l'exigence de dédier les objectifs de la production scientifique à la résolution des problèmes industriels considérés comme les seuls dignes d'intérêt pour la collectivité. Elle s'accompagne d'un dénigrement du politique, des arts, du travail intellectuel en général, de la futilité de l'abstraction face à l'action tournée vers le productivisme. C'est une exaltation permanente de l'action opposée à la pensée qui ne serait pas conduite par la science ${ }^{32}$. La position se traduit, dans son expression la plus franche, par la proposition de substituer un corps de scientifiques aux élus pour la conduite rationnelle de la société moderne. C'est une proposition formulée dans le rapport livré par Étienne Clémentel en 1919, mais que l'on retrouve dans nombre de ses écrits d'après guerre. Henry Le Chatelier multiplie à l'évidence les efforts pour parvenir à une institutionnalisation du pouvoir économique et politique par les scientifiques.

\footnotetext{
${ }^{29}$ Armand Hatchuel, «Frederick Taylor : une lecture épistémologique. L'expert, le théorcien, le doctrinaire », dans J. P. Bouilloud et B. P. Lecuyer, dir. L'invention de la gestion. Histoires et pratiques, Paris, L'Harmattan, 1994, p. 53-64.

${ }^{30}$ Luc Rouban, L'État et la science. La politique publique de la science et de la technologie, Paris, Éditions du CNRS, 1988, p. 56.

${ }^{31}$ Richard Déry, «Enjeux et controverses épistémologiques dans le champ des sciences de l'administration », Revue canadienne des sciences de l'administration, 9, 1992, p. 1-12.

${ }^{32}$ Henry Le Chatelier, «Quelques problèmes scientifiques à résoudre », Comptes rendus de l'Académie des sciences, 164, 1917 , p. 205-210.
} 
La contestation du politique par Henry Le Chatelier pose la question récurrente en France d'une rationalité des politiques publiques pour lesquelles il faudrait et il suffirait d'appliquer au système politico-administratif les règles, les principes et les méthodes de la démarche scientifique. D'où la vision exaltée chez Henry Le Chatelier d'une société qui substitue à la conscience de classe les fonctions des groupes sociaux et la volonté d'optimiser, à l'aide des sciences sociales appliquées, l'orientation professionnelle des agents et le rendement social de chacun. De la science industrielle et de la rationalisation selon Henry Le Chatelier se dégage dès lors le modèle technocratique de gestion des relations entre la science et la production, la science et l'organisation, la science et la politique, avec pour figure centrale celle de l'ingénieur scientifique, organisateur et rationalisateur. Ce dernier, par sa formation, ses compétences et ses fonctions sociales est idéalement situé pour combattre l'idée subversive à ses yeux d'une subordination de la science à la politique. La prétention est d'instaurer l'ordre nécessaire dans une société hiérarchisée par les scientifiques et les ingénieurs, détenteurs légitimes du savoir et de la raison pratique, seuls autorisés à valider les impératifs d'action orientée vers l'organisation rationnelle d'une société industrielle.

\section{Corpsards et culte de la rationalisation.}

Certes les certitudes scientistes et déterministes telles que les exprime Henry Le Chatelier n'appartiennent qu'à lui. Il n'est, après tout, que l'un des membres d'une communauté d'acteurs de la rationalisation qui exalte sans complexe les vertus du centralisme technocratique. Le culte de la science et de la technique, la foi dans le progrès industriel ne sont à l'évidence pas l'apanage des corpsards des Mines. Henry Le Chatelier n'en demeure pas moins une figure emblématique de cette culture de la rationalisation à la française confinant au culte de la rationalisation. Ses liens avec le statut même de l'ingénieur d'État, avec la culture, les pouvoirs et les pratiques propres au corps des Mines ne peuvent être ignorés. Nul autre ingénieur n'est en effet aussi fermement et si tôt assuré de sa fonction d'éducateur de la Nation industrielle. Le corpsard des Mines, du fait de son recrutement social et la sélection sévère par les mathématiques à laquelle il a dû se soumettre, de sa formation, de la place qu'il occupe au sein de l'appareil étatique, du fait de son statut d'aristocrate des savoirs rationnels et l'attribution enfin d'un monopole sur la science et les technologies, affirme son incontestable mérite et sa supériorité. Il prolonge et consolide un État dans sa composante la plus technocratique, dont l'École polytechnique est l'institution de référence, la base principale de sa constitution $^{33}$. Il est par excellence l'agent de la bureaucratisation et renforce tant l'autorité de l'État que le pouvoir de la classe dirigeante dont il partage objectivement les vues et les intérêts.

Son champ d'intervention demeure toutefois la réalité de la production industrielle auprès de laquelle il exerce un rôle à la fois de pédagogue et de supervision administrative. Il lui arrive aussi d'assurer la direction même des établissements sur lesquels il a exercé un temps le contrôle ${ }^{34}$. De sorte que les liens entre production scientifique et pratique industrielle ne sont pas seulement ceux d'experts désignés par l'État afin d'assurer la gestion administrative du secteur privé au profit de l'intérêt général. Cette relation fondatrice du corps des Mines se traduit aussi par l'importation en retour de modèles d'organisation et de gestion des administrations publiques calqués sur ceux de l'entreprise.

L'ingénieur des Mines n'est toutefois pas que l'exécutant d'un dessein général visant la rationalisation de la société industrielle. Il forme une élite intellectuelle, participe activement au mouvement des idées, investi parfois le débat public. Même s'il ne s'exprime qu'avec parcimonie dans l'arène politique, tente de respecter une forme de neutralité et se contente généralement de servir loyalement les régimes successifs, une majorité d'entre eux opte pour la République plus encline à exalter le progrès par les sciences, et plus particulièrement sous la $\mathrm{III}^{\mathrm{e}}$ République $^{35}$. Ils sont ainsi vingt au total à avoir siégé au Parlement ou au Sénat depuis la création du corps des Mines jusqu'à nos jours : Jean Bineau, Michel Chevalier, Eutrope-Barthélémy de Cressac, Louis Hericart de Thury, François Loos, Félix de Martha-Becker, Jean-Louis Masson, Jean Reynaud, Lionel Stoleru, Félix

\footnotetext{
${ }^{33}$ Bruno Belhoste, La formation d'une technocratie. L'École polytechnique et ses élèves de la Révolution au Second Empire, Paris, Belin, 2003, 507 p.

34 Terry Shinn, «Des corps de l'État au secteur industriel : genèse de la profession d'ingénieur, 1750-1920 », Revue française de sociologie, Paris, Ophys et Association Revue française de sociologie, 1978, p. 39-71.

${ }^{35}$ Terry Shinn, L'École polytechnique : 1794-1914, Paris, Presses de la Fondation nationale des sciences politiques, 1980 , $261 \mathrm{p}$.
} 
Varin, Jacques Vernier, dont neuf sous la seule III $^{\mathrm{e}}$ République : Eloi Beral, Aimé Blavier, Charles Freycinet, Léon Janet, Albert Lebrun, François Sauvage, Édouard Sens, Mercal Ulrich et Charles Wickesheimer. Le bilan reste néanmoins que les ingénieurs des Mines n'intègrent ni plus ni moins que les autres catégories d'ingénieurs les instances gouvernementales ou n'embrassent la carrière politique aux côtés d'un parti en particulier ${ }^{36}$.

Le mode d'intervention de l'ingénieur privilégie en réalité la production plus discrète des outils de la rationalité instrumentale mis à la disposition des acteurs de l'organisation et de la réforme sociale. Il initie ou participe aux travaux des laboratoires de la pensée, à l'élaboration des programmes d'action livrés par les annexes du pouvoir législatif et de l'exécutif, par la multitude des associations, sociétés ou organisations dont les vocations sont d'influencer pour les façonner les institutions et les politiques sociales. Le mouvement saint-simonien, le positivisme, la réforme sociale et l'économie industrielle comptent ainsi parmi ses instigateurs et adeptes de nombreux corpsards. Michel Chevalier ou Frédéric Le Play restent les éternelles figures emblématiques. Leurs disciples et héritiers ont cependant participé au tournant du siècle à la gestion, pour parfois s'y opposé, au consensus libéral et démocratique de la société de masse. Les ingénieurs des Mines ont ainsi contribué à l'idée qu'une science des sociétés était possible et ont travaillé à l'essor des sciences sociales ${ }^{37}$.

$\mathrm{Au}$ terme de cette incursion dans l'univers de la rationalisation chez les ingénieurs, et chez Henry Le Chatelier en particulier, peut-on associer aux pouvoirs et aux pratiques des corpsard des Mines une culture spécifique ? Le culte de la rationalisation peut bien ainsi devoir caractériser plus précisément un mode d'être au monde social, un état d'esprit, et singulariser les justifications de leurs actions. Il se fonde en grande partie sur la conviction de détenir des savoirs spécifiques justifiant leur fonction d'organisateur, et que les textes fondateurs du corps et de la législation de 1810 leur ont assigné : produire des instruments de mesure de la réalité industrielle, économique et sociale ; élaborer les moyens de son contrôle et proposer des guides pour l'action dans le domaine de l'intervention publique. Sa sphère d'action se situe au croisement de l'administration, de la science et de l'industrie ; de l'organisation, de la théorie et de la pratique. Elle se traduit par l'exercice d'une tutelle sur la collectivité au nom du verdict de la raison comme critère principal de légitimité de toute institution humaine ${ }^{38}$.

Par l'attribution de ses fonctions qui le lient au devenir même de l'État, l'ingénieur des Mines renvoie, plus que tout autre fonctionnaire et membre de la communauté des élites, à l'idéal typique de l'administrateur en charge de la rationalisation de la production et du développement industriel de la Nation. Leurs prérogatives préludent au $19^{\mathrm{e}}$ siècle à l'émergence des commis d'un État rationalisateur et modernisateur dont l'autorité est celle de la Raison. Il annonce au suivant l'agent de la bureaucratisation et la figure du technocrate planificateur ${ }^{39}$. C'est du côté de ces domaines d'intervention de l'ingénieur des Mines qu'il faut sans doute rechercher les traits distinctifs d'une culture de la rationalisation, érigée parfois en culte de la rationalisation chez nombre d'entre eux.

\footnotetext{
${ }^{36}$ Bruno Marnot, Les ingénieurs au Parlement sous la III République, Paris, CNRS éditions, 2000, 322 p.

${ }^{37}$ Antoine Savoye et Frédéric Audren, «Les ingénieurs des mines et les sciences sociales au XIX ${ }^{\mathrm{e}}$ siècle : le filon leplaysien », dans Antoine Savoye et Frédéric Audren, dir. Naissance de l'ingénieur social. Les ingénieurs des mines et la science sociale au XIX ${ }^{e}$ siècle, Paris, Presses ENSMP, 2008, pp. 9-22; Bernard Kalaora et Antoine Savoye, Les inventeurs oubliés : Le Play et ses continuateurs aux origines des sciences sociales, Seyssel, Champ Vallon, $293 \mathrm{p}$.

${ }^{38}$ André Thepot, Les ingénieurs des mines du XIX ${ }^{e}$ siècle. Histoire d'un corps technique d'État. Tome 1: 1810-1914, Paris, Éditions Eska, 1998, 511 p.

${ }_{39}$ Pierre Rosanvallon, L'État en France, de 1789 à nos jours, Paris, Seuil, 1990, p. 221-225.
} 